\title{
Le récepteur de la leptine appartient à la famille des récepteurs de cytokines
}

Un an à peine après le clonage du gbne $o b$ par l'équipe de J. Friedman (voir $\mathrm{m} / \mathrm{s}, n^{\circ} 12$, vol. 11, p. 1337), les gènes murin et humain d'un récepteur de la leptine (produit du gène $o b)$ viennent d'être isolés par des chercheurs de Millennium Pharmaceutical (Cambridge, MA, USA) et de Hoffman-La Roche (Gand, Belgique, Nutley, NJ, USA et Bâle, Suisse) [1]. Le clonage de ce récepteur intervient dans un contexte où il apparaît de plus en plus évident que la grande majorité des obèses ne sont pas déficients en leptine, comme c'est le cas de la souris $o b / o b$, mais au contraire en produisent de grandes quantités $\left(\mathrm{m} / \mathrm{s} n^{\circ} 2\right.$, vol. 12, p. 257). Or, le pouvoir amaigrissant de la leptine a été démontré sans ambiguité chez la souris [2]. Bien que l'on ne puisse pas exclure totalement que la leptine n'exerce pas les mêmes fonctions chez l'homme et chez les rongeurs, l'hypothèse la plus plausible est qu'il existe des états de résistance à la leptine dont la base moléculaire pourrait se situer dans la voie de transmission du signal relayant les effets biologiques de l'hormone. D'où l'intérêt de la découverte de son récepteur, étape préalable indispensable à l'étude de la transmission du signal. La disponibilité de la protéine recombinante a permis un clonage d'expression du récepteur. Une banque d'ADN complémentaires, préparée à partir de plexus choroïde de souris au niveau duquel une forte liaison de leptine avait été détectée, a été exprimée dans des cellules COS. Les différents clones obtenus ont été testés par groupes pour la liaison de la leptine, jusqu'à obtention d'un seul clone positif. L'ADNc présent dans ce clone est une séquence de
$5,1 \mathrm{~kb}$ constituant un cadre de lecture unique qui code pour une nouvelle protéine de 894 acides aminés, OB-R. La protéine mûre serait composée d'une longue chaîne extracellulaire de 816 acides aminés, d'un domaine transmembranaire (23acides aminés) et d'un court domaine cytoplasmique de 34 acides aminés. Ce qui est particulièrement intéressant est que OB-R présente des caractéristiques de récepteur de cytokine qui le rapprochent de la chaîne gp 130 du récepteur de l'interleukine 6 , du récepteur du LIF (leukemia inhibitory factor) et du récepteur du G-CSF (granulocyte colony stimulating factor). La transduction du signal par ce type de récepteur est partiellement décryptée [3]. Elle met en jeu la formation de complexes multimériques de différentes chaînes, ce qui conduit au recrutement de tyrosine kinases, en particulier celles de la famille JAK, qui vont phosphoryler des facteurs de transcription de type Stat [3]. Les gènes cibles de ces facteurs sont encore mal connus. D'autres voies, en particulier la voie de Ras et des MAP kinases, semblent également pouvoir intervenir dans la transmission du signal par les cytokines. La découverte d'un récepteur type cytokine pour la leptine va donc cibler les recherches futures visant à analyser les voies de transmission du signal de cette hormone. Il faut noter qu'une recherche systématique d'homologie de structure tridimentionnelle a permis récemment de montrer que la séquence de la leptine était compatible avec une conformation de cytokine [4].

L'analyse de la distribution tissulaire de l'ARNm de $O B-R$ révèle une expression relativement ubiquiste, avec un signal fort dans le poumon et le rein et des signaux plus faibles dans d'autres tissus, cerveau, foie, cœur, muscles squelettiques, etc. Dans le cerveau, l'amplification par RT-PCR de l'ARNm a permis de mettre en évidence l'expression de $O B-R$ dans l'hypothalamus, en bon accord avec la démonstration antérieure d'une liaison de leptine à des membranes plasmiques d'hypothalamus de rat [5]. L'importance de l'hypothalamus dans la régulation de la balance énergétique est bien connue, ce qui laisse penser que la liaison de leptine à ce niveau joue un rôle crucial dans la fonction biologique de l'hormone. En particulier, il est possible que la leptine puisse moduler l'action de neuropeptides régulateurs de la prise alimentaire. Ainsi, il a été montré que la leptine diminue la synthèse et la sécrétion du neuropeptide hypothalamique NP-Y, puissant stimulateur de l'appétit [5]. Elle pourrait également interagir avec le GlucagonLike Peptide 1 (GLP-1), dont on sait depuis peu qu'il se lie au niveau de l'hypothalamus et exerce un fort effet de satiété chez le rat [6]. Quel est le rôle des OB-R présents dans les autres parties du cerveau et dans les tissus périphériques? Pour l'instant cette question est sans réponse.

Il reste une inconnue quant à la fonction exacte de la protéine découverte. Le récepteur murin cloné à partir du plexus choroïde est-t-il capable de transmettre un signal ? Il pourrait, en fait, s'agir d'un transporteur de leptine, facilitant son passage à travers la barrière hématoméningée. Il faut également remarquer que le récepteur humain cloné par les mêmes auteurs présente un domaine intracellulaire beaucoup plus long que le récepteur murin. S'agit-il de deux 
formes de la même protéine résultant d'un épissage alternatif? Les deux formes sont-elles exprimées spécifiquement dans différents tissus ou suivant l'espèce? Jouent-elles des rôles différents dans la transmission du signal leptine? Il est certain que des réponses seront apportées à ces questions dans un avenir proche.

L'une des hypothèses anciennes réactualisées par la découverte de la leptine est qu'un récepteur ou un effecteur de cette hormone serait codé par le gène $d b$. La question s'est donc immédiatement posée de savoir si OB-R était le produit du gène $d b$. En faveur de cette hypothèse, une cartographie génétique du gène $O B-R$ le situe dans l'intervalle de $5,1 \mathrm{cM}$ du chromosome 4 de la souris contenant le locus $d b$. Cependant, l'ARNm de OB-R est détecté dans le plexus choroïde et l'hypothalamus des souris $d b / d b$, pratiquement au même niveau d'expression que chez les témoins et aucune muta- tion n'a été trouvée dans sa partie codante. Enfin, une liaison de leptine est mesurable dans le plexus choroïde des mutants. Ces observations suggèrent que la mutation $d b$ n'entraîne pas de perte d'expression de OB-R. On peut cependant émettre l'hypothèse que cette mutation affecte une autre forme de OB-R, peut être celle présentant le long domaine intracellulaire qui pourrait être plus spécifiquement impliquée dans la transmission du signal dans certains tissus.

Finalement, la découverte d'un récepteur de leptine ouvre de nouveaux champs de recherche pharmaceutique vers des substances capables d'augmenter l'efficacité biologique de la leptine ou même de mimer ses effets. De telles molécules, si elles existent, pourraient révolutionner le traitement de certaines obésités.

M.G.-M.
1. Tartaglia LA, Dembski M, Weng X, Deng N, Culpepper J, Devos R, Richards GJ, Campfield LA, Clark FT, Deed J, Muir C, Sanker S, Moriarty A, Moore KJ, Smutko JS, Mays GG, Woolf EA, Monroe CA, Tepper RI. Identification and expression cloning of a leptin receptor, OB-R. Cell $1995 ; 83: 1263-71$.

2. Kahn A. Une confirmation: le produit du gène $o b$ est bien une hormone agissant comme un lipostat. médecine/sciences $1995 ; 11: 1463-4$.

3. Dusanter-Fourt I, Mayeux P, Gisselbrecht S. Transduction du signal par les récepteurs de cytokines. médecine/sciences 1994; 10 : 825-35.

4. Madej T, Boguski MS, Bryant SH. Threading analysis suggests that the obese gene product may be a helical cytokine. FEBS Lett 1995 ; 373 : 13-8.

5. Stephen TW, Basinski M, Bristow PK, Bue-Valleskey JM, Burgett SG, Craft L, Hale J, Hoffmann J, Hsiung HM, Kriauciunas A, MacKellar W, Rosteck Jr PR, Schoner B, Smith D, Tinsley FC, Zhang XY, Heiman M. The role of neuropeptide $\mathrm{Y}$ in the antiobesity action of the obese gene product. Nature $1995 ; 377: 530-2$.

6. Turton MD, O'Shea D, Gunn I, Beak SA, Edwards CMB, Meeran K, Choi SJ, Taylor GM, Heath MM, Lambert PD, Wilding JPH, Smith DM, Ghatei MA, Herbert J, Bloom SR. A role for glucagon-like peptide-1 in the central regulation of feeding. Nature 1996; 379: 69-72. 